\title{
BRDF Reconstruction from Real Object using Reconstructed Geometry of Multi-view Images
}

\author{
Taishi Ono \\ Nara Institute of Science and Technology \\ Takuya Funatomi \\ Nara Institute of Science and Technology
}

\author{
Hiroyuki Kubo \\ Nara Institute of Science and Technology \\ Yasuhiro Mukaigawa \\ Nara Institute of Science and Technology
}

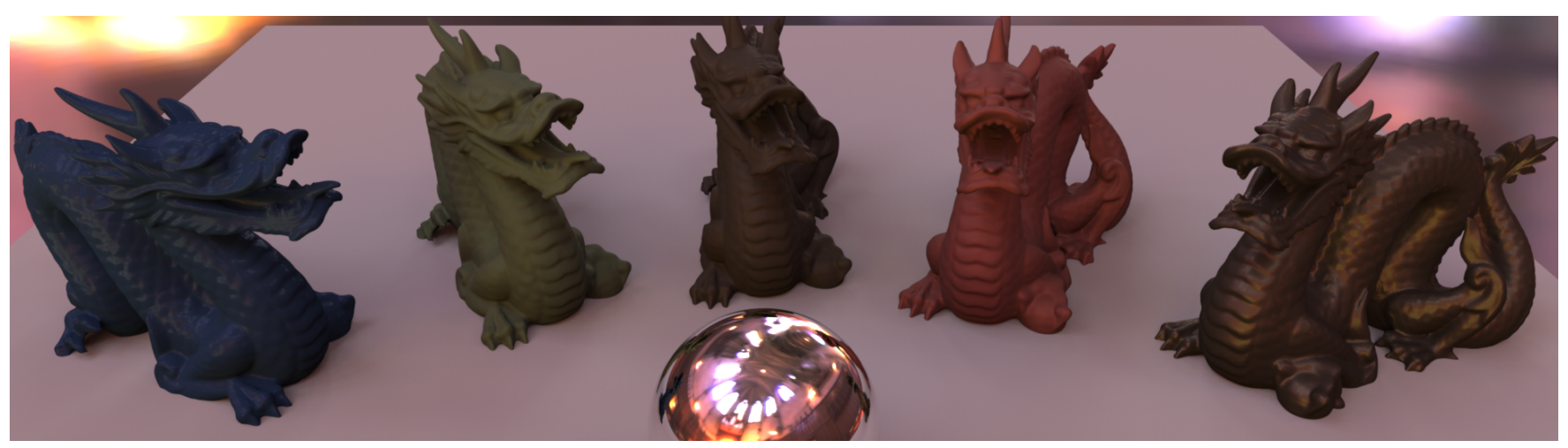

Figure 1: Rendering of the reconstructed BRDFs using our method.

\begin{abstract}
In this paper, we present an effective approach to reconstruct the bidirectional reflectance distribution function (BRDF) from a real object without any special devices and complex setups. Multi-view images are commonly used to reconstruct the three-dimensional (3D) shape of an object, and this is known as photogrammetry. For the purpose of photo-realistic rendering of an object, our approach is practical because it is highly integrated into the photogrammetry pipeline. Our method requires to acquire multi-view images for $3 \mathrm{D}$ reconstruction and a few additional images to obtain the reflectance distribution. We use the reconstructed geometry from multi-view images to determine the region of optimal light and view directions according to the existing analytic methods. However, the reconstructed geometry and surface normal tend to contain artifacts. Therefore, we introduced assumptions that are the main contribution of our study to extract the reliable regions from the reconstructed geometry. The results demonstrate that our method effectively acquires a plausible BRDF.
\end{abstract}

\section{CCS CONCEPTS}

- Computing methodologies $\rightarrow$ Appearance and texture representations;

(c) (i) $\Theta$ :
This work is licensed under a Creative Commons
Attribution-NonCommercial-NoDerivs International 4.0 License

SA '17 Technical Briefs, November 27-30, 2017, Bangkok, Thailand

(c) 2017 Copyright held by the owner/author(s).

ACM ISBN 978-1-4503-5406-6/17/11.

https://doi.org/10.1145/3145749.3149426

\section{KEYWORDS}

BRDF reconstruction, multi-view images

ACM Reference format:

Taishi Ono, Hiroyuki Kubo, Takuya Funatomi, and Yasuhiro Mukaigawa. 2017. BRDF Reconstruction from Real Object using Reconstructed Geometry of Multi-view Images. In Proceedings of SIGGRAPH Asia 2017 Technical Briefs, Bangkok, Thailand, November 27-30, 2017 (SA '17 Technical Briefs), 4 pages. https://doi.org/10.1145/3145749.3149426

\section{INTRODUCTION}

Recently, automatic three-dimensional (3D) shape reconstruction from multi-view images has become more popular. These reconstructed 3D objects are used in many movies and video games because of their highly detailed geometry. However, the appearance is not sufficiently plausible because the reflectance distribution property is not observed from the target object.

In this paper, we present an effective approach to BRDF reconstruction from a real object without any special devices or complex setups. We use multiple-images that are originally prepared for the photogrammetry, and a few additional images for BRDF acquisition. Prior to obtaining the reflectance property, we reconstruct the 3D shape from multi-view images, however, the reconstructed shape tends to contain geometric artifacts. Therefore, we determine the reliable region which contains less artifact of optimal light and view directions according to the method of [Nielsen et al. 2015]. Our main contribution is to introduce a few assumptions to extract the reliable region of the reconstructed geometry. The advantage of our work is its practicability because our approach is highly integrated into the existing photogrammetry pipeline. 


\section{RELATED WORK}

There are a lot of researches on reconstructing the geometry of an object from multiple-images. Chandraker et al. [2013] acquired the object's shape from its motion with unknown isotropic BRDF. They proved that four different motions are sufficient to yield the depth and surface gradient.

To reconstruct the 3D shape and BRDF from a real object, Müller et al. [2005] developed a measurement device that used camera and light arrays. However, BRDF sampling requires millions of measurements. Therefore, these methods are not practical because they need a great number of expensive devices and complex setups.

Focusing on the sampling costs, Nielsen et al. [2015] significantly reduced the number of samples to recounstruct BRDF. They extracted the principal components of the set of BRDFs from the MERL BRDF database [Matusik et al. 2003] which contains 100 real BRDFs, and optimized the sampling directions to minimize the condition number of the related acquisition matrix. One of the disadvantages is that the method can only be applied to the known shape geometry and homogeneous object because the method requires the accurate surface normal direction of the object.

In this paper, we introduce a method to obtain both the shape and BRDF from multi-view images of the real object.

\section{BRDF RECONSTRUCTION}

Figure 2 shows the overview of our method. Prior to reconstructing the BRDF, we obtain multi-view images in ambient light (Fig. 3(a)), and then, reconstruct the geometry from these images. This process is the photogrammetry pipeline. We also acquire a few images with a single distant light, as shown in Fig. 3(b). From these images, we determine regions of optimal light and view directions to reconstruct the BRDF. In the following subsection, we briefly describe the process for our approach.

\subsection{Reliable Region Extraction to Avoid the Reconstruction Artifact of the Normal Direction}

To estimate the BRDF accurately, it is necessary to prepare the accurate normal direction of the object. Figure 3(c) shows the normal map of the reconstructed geometry and Figure 3(d) shows the error map of the angle between the reconstructed and ground truth normal direction. This shows that several regions of the reconstructed normal direction contain the artifact, unfortunately. If we ignore these errors of the surface normal, the reconstructed BRDF will also be significantly inconsistent with the ground truth, as shown in Fig. 3(e).

Thus, we introduce a few assumptions to extract the reliable region from the reconstructed geometry. We assume that the error of the surface normal has a strong correlation with the curvature of the reconstructed object because the reconstructed geometry using the multi-view images tends to be blunted. This means that the normal direction of the high curvature region is not reliable. We also assume that the reflectance distribution should satisfy the monotonicity with respect to the cosine between the surface normal and half vector. This assumption is introduced in [Higo et al. 2010], which describes a photometric stereo that works with a wide range of BRDFs. Finally, we also assume that the highest intensity region represents the peak of the specular reflection. In our experiment, we implement these assumptions as constraints using the following steps:

\section{- Curvature}

First, we calculate the principal curvatures $\kappa_{u}$ and $\kappa_{v}$ which correspond to principal directions $\mathbf{u}$ and $\mathbf{v}$ in the image space proposed by Vergne et al. [2009]. We use $H=\left(\kappa_{u}+\kappa_{v}\right) / 2$ as the curvature of each pixel. Finally, we remove unreliable pixels that had a high curvature according to the threshold.

\section{- Monotonicity}

Given the lighting direction $\mathbf{l}$, view direction $\mathbf{v}$, surface normal $\mathbf{n}$, and half vector $\mathbf{h}=\frac{\mathbf{l}+\mathbf{v}}{\|\mathbf{l} \mathbf{v}\|}$, we assume that BRDF $r$ at pixels $i$ and $j$ satisfies the following equation:

$$
\mathbf{n}_{i} \cdot \mathbf{h}_{i}>\mathbf{n}_{j} \cdot \mathbf{h}_{j} \Leftrightarrow r_{i}>r_{j}
$$

We avoid using the unreliable pixels, which does not satisfy Eq. (1) for BRDF reconstruction.

- Region with the highest intensity

For the region $k$ with the highest intensity, we assume that the directions of the half vector $\mathbf{h}$ and surface normal $\mathbf{n}$ are completely equal, which is explained by the following equation.

$$
\mathbf{n}_{k} \cdot \mathbf{h}_{k}=1
$$

We determine the pixel with the highest intensity among all pixels in the distant light images, and then modify its surface normal to satisfy Eq. (2).

\subsection{Choosing Optimal Pixels}

After extracting reliable region using these assumptions, we determine the appropriate pixels for the sampling directions according to the method of [Nielsen et al. 2015]. We determine pixels in the reliable region to minimize the condition number of the acquisition matrix obtained by [Nielsen et al. 2015] and reconstruct the BRDF from these pixels.

Even under the proposed constraints, the normal directions of several pixels are inaccurate. Therefore, we randomly extract pixels from the reliable region and execute BRDF reconstruction several times. We render images using the reconstructed shape and the reconstructed BRDF from the same viewpoint as the captured images. We acquire the KL divergences of the intensity distribution between these images, and define the reconstruction with the minimum KL divergence as the most plausible result.

\section{EXPERIMENTAL RESULTS}

We demonstrate the effectiveness of our method in simulated environments and also apply this method to real objects.

\subsection{Simulation}

We executed four simulations on the MERL BRDF database and the fully measured geometry (Stanford Bunny and Dragon, by Stanford Computer Graphics Laboratory) as the ground truth.

For this simulated experiment, Figure 3(a) shows one of the rendered multi-view images for $3 \mathrm{D}$ reconstruction. In this case we rendered 55 images with $3,000 \times 2,000 \mathrm{px}$ resolution rendered by pbrt[Pharr and Humphreys 2010]. These images covered spherically of the target object. Figure 3(b) shows one of the images 


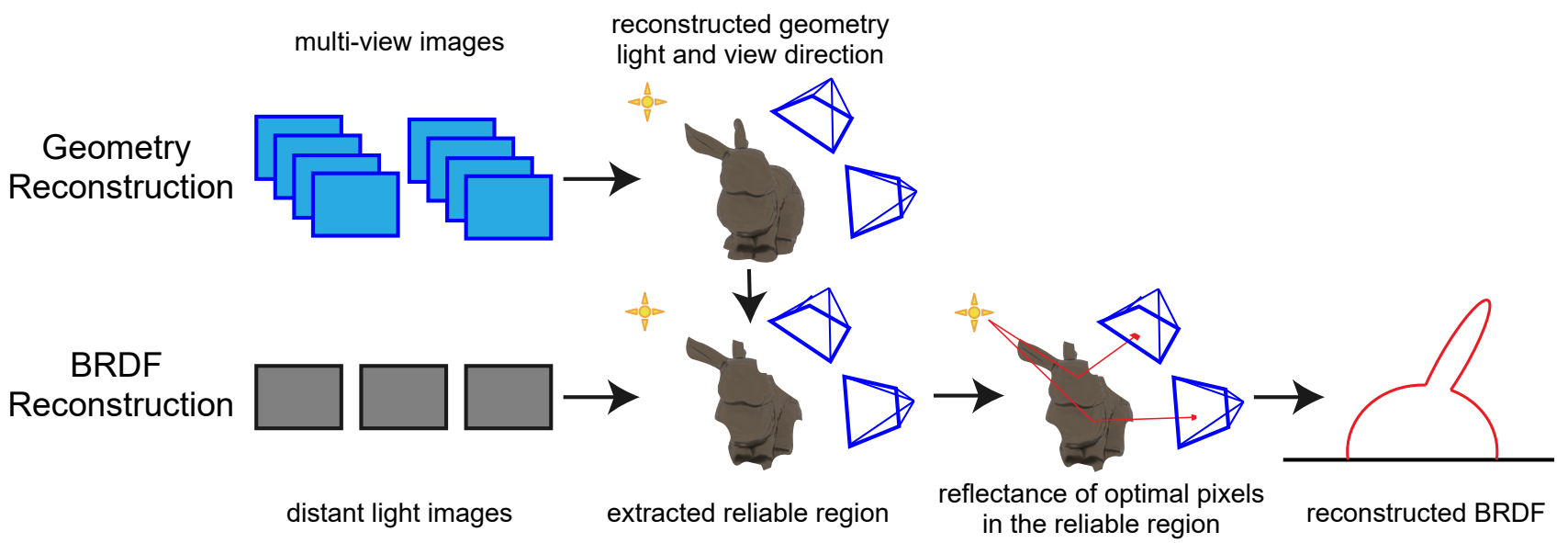

Figure 2: Overview of our method. First we acquire multi-view images with ambient light, and also acquire a few images with only one distant light. Next, we reconstruct the 3D shape and estimate the light and view directions of multi-view images. Then we analytically determine the reliable region of optimal light and view directions, and obtain the BRDF at the corresponding pixels. Finally, we acquire a plausible BRDF.

rendered with a virtual single distant light. These images were used to calculate the BRDF at chosen pixels. In this case, we used 11 images for BRDF reconstruction. Each of these images was rendered from the same viewpoint as one of 55 multi-view images. For the 3D reconstruction, we used Agisoft Photoscan (Agisoft LLC, www.agisoft.com). In this case, we set the threshold of the curvature to 0.2 , and used six pairs of lights and view directions as the optimal directions for BRDF reconstruction.

Figure 4 shows the results of reconstructed BRDFs. The graphs on the left column show the comparison between the reconstructed BRDFs and ground truth. The pictures on the right column show the rendered images using the reconstructed BRDFs and ground truth. In comparison with Fig. 3(e), we observe a noticeable improvement in reconstruction quality, in particular around the specular direction. Additionally, the appearance of both the rendered images using the reconstructed BRDFs and ground truth BRDFs seems to be quite similar. Figure 1 shows the rendered image of the reconstructed BRDFs with detailed geometry and environment lighting (Grace Cathedral, (1)1998, 1999 Paul Debevec [Debevec 1998]). This also indicates that our method can acquire the plausible appearance.

\subsection{Real Environment}

We applied our method to real objects. In this case, we reconstructed the BRDF of a hammer. The experimental setup is shown in Fig. 5. We used an SLR camera (Nikon D5500) to capture images and SIGMAKOKI SLA-100A as the distant light. We obtained 73 multiview images under room lighting for $3 \mathrm{D}$ reconstruction and eight images with the distant light. Using a clear coat black sphere, we estimated the direction toward the distant light. In this experiment, we regarded the hammer as composed of two materials, thus, we individually reconstructed the BRDF from the head and body of the hammer. Figure 6 shows the rendered images with the reconstructed BRDF from the head and body of the hammer.

We also reconstructed BRDF of an object located in outdoor, as shown in Fig. 7. In this case, We assumed the sunlight direction

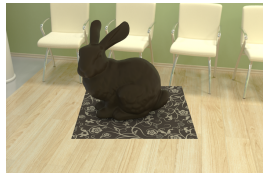

(a)

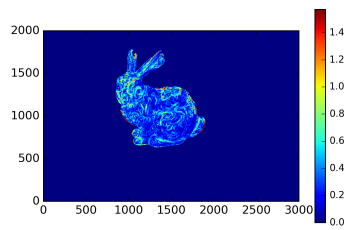

(d)

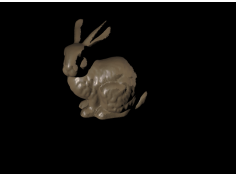

(b)

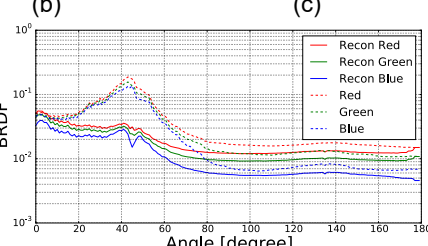

(e)
Figure 3: (a) One of the multi-view images used for 3D reconstruction. (b) One of the images rendered with a distant light for acquiring the BRDF at optimal pixels. (c) Reconstructed normal map. (d) Relation of the angle[rad] between reconstructed surface normal and ground truth. (e) Comparison between the reconstructed BRDFs without any constraints(solid) and ground truth (dashed).

by the time and place of pictures. We separate the pole into two areas, the central metallic area and the other parts, and individually reconstructed BRDF. Figure 7 shows the rendered images with the reconstructed BRDF. Our method can work well even in the outside, and this also represents the practicability of our method because photogrammetry is often applied to outside objects. In each experiment, the reconstructed appearance is plausible.

\section{CONCLUSION AND FUTURE WORK}

In this paper, we developed a truly practical method of BRDF acquisition, which is highly integrated with the production pipeline of photogrammetry, that required a few distant light images in addition to multi-view images for $3 \mathrm{D}$ reconstruction. The main 

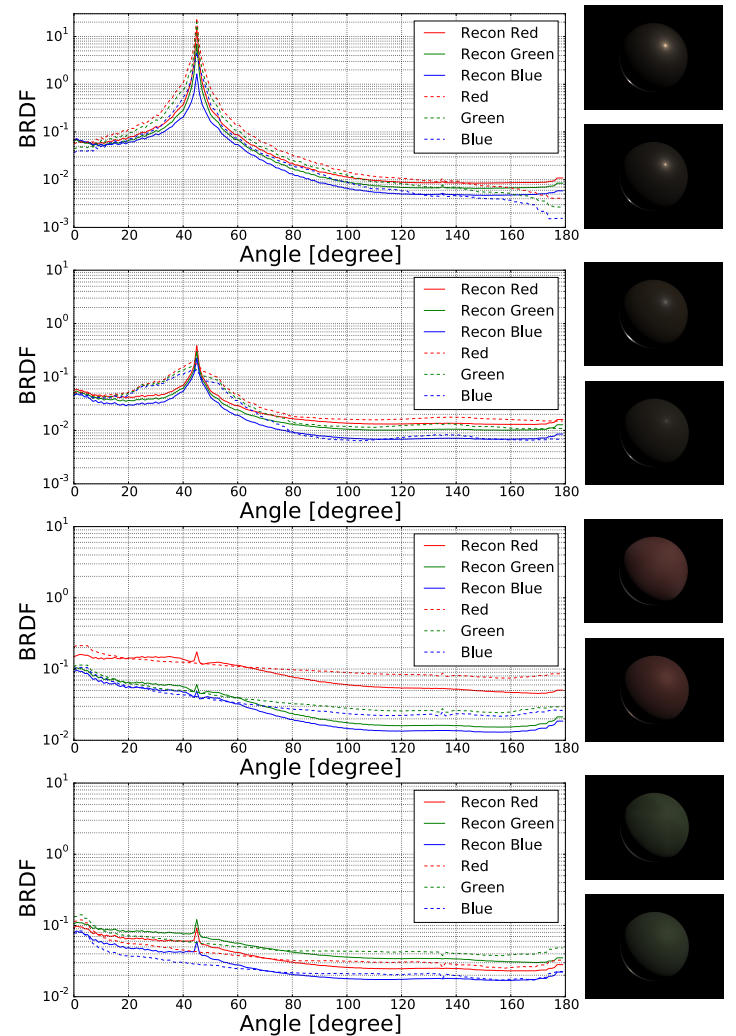

Figure 4: Reconstructed BRDF for four simulations: alumbronze, fruitwood-241, pink-fabric2, and green-latex from the MERL BRDF database. Left: Comparison between the reconstructed BRDFs (solid) and ground truth (dashed). Incident light is at 135 degrees. Upper right: Rendered sphere with ground truth. Lower right: Rendered sphere with reconstructed BRDFs.
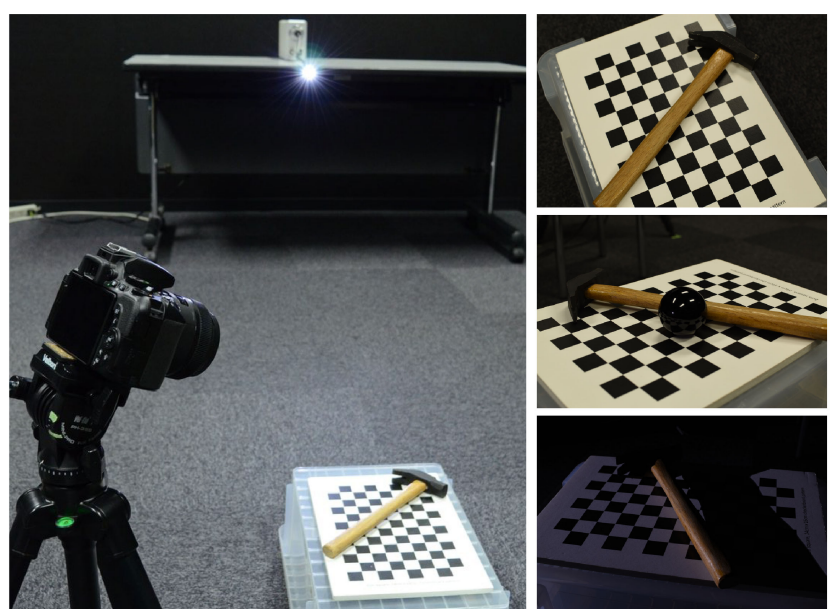

Figure 5: Left: Experimental setup. Right upper: One of the multi-view images for 3D reconstruction. Right middle: Picture with black sphere for obtaining the light direction. Right lower: One of the distant light images
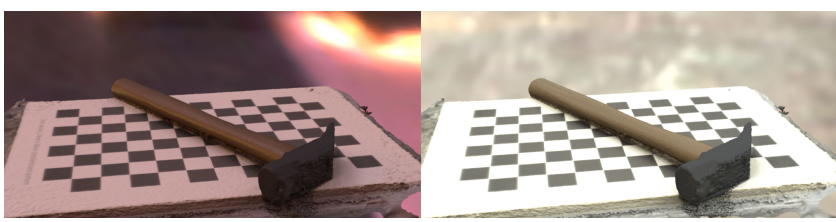

Figure 6: Rendered images using the reconstructed BRDF with two environment lightings (๑1998, 1999 Paul Debevec).

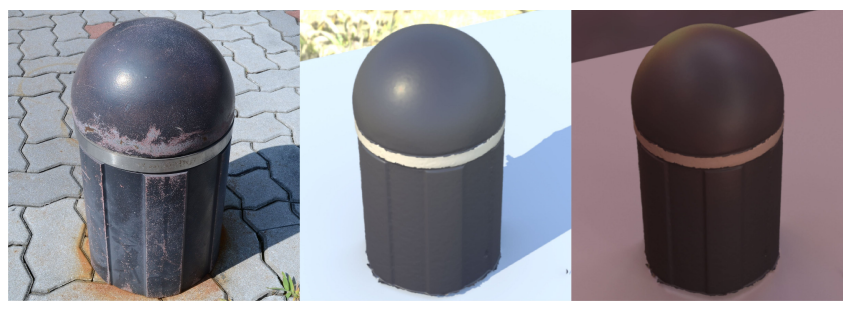

Figure 7: Left: One of the input multi-view images. Middle and Right: Rendered images with reconstructed BRDF.

technical contribution is to introduce some assumptions to extract more reliable regions and reconstruct a more accurate BRDF, and our results show that these constraints provide plausible outputs.

To reconstruct a more accurate BRDF from a real object, we should consider the textures of materials. We should also discuss the number of sampling points and threshold of curvature in more detail because, currently, we empirically determine these values.

\section{ACKNOWLEDGEMENTS}

This work is partly supported by JSPS KAKENHI JP15K16027, JP26700013 and JP15H05918.

\section{REFERENCES}

M. Chandraker, D. Reddy, Y. Wang, and R. Ramamoorthi. 2013. What Object Motion Reveals about Shape with Unknown BRDF and Lighting. In 2013 IEEE Conference on Computer Vision and Pattern Recognition. 2523-2530. https://doi.org/10.1109/ CVPR.2013.326

Paul Debevec. 1998. Rendering Synthetic Objects into Real Scenes: Bridging Traditional and Image-based Graphics with Global Illumination and High Dynamic Range Photography. In Proceedings of the 25th Annual Conference on Computer Graphics and Interactive Techniques (SIGGRAPH '98). ACM, New York, NY, USA, 189-198. https://doi.org/10.1145/280814.280864

T. Higo, Y. Matsushita, and K. Ikeuchi. 2010. Consensus photometric stereo. In 2010 IEEE Computer Society Conference on Computer Vision and Pattern Recognition. 1157-1164. https://doi.org/10.1109/CVPR.2010.5540084

Wojciech Matusik, Hanspeter Pfister, Matthew Brand, and Leonard McMillan. 2003. Efficient Isotropic BRDF Measurement. In Proceedings of the 14th Eurographics Workshop on Rendering (EGRW'03). Eurographics Association, Aire-la-Ville, Switzerland, Switzerland, 241-247. http://dl.acm.org/citation.cfm?id=882404.882439

Gero Müller, Gerhard H. Bendels, and Reinhard Klein. 2005. Rapid Synchronous Acquisition of Geometry and Appearance of Cultural Heritage Artefacts. In Proceedings of the 6th International Conference on Virtual Reality, Archaeology and Intelligent Cultural Heritage (VAST'05). Eurographics Association, Aire-la-Ville, Switzerland, Switzerland, 13-20. https://doi.org/10.2312/VAST/VAST05/013-020

Jannik Boll Nielsen, Henrik Wann Jensen, and Ravi Ramamoorthi. 2015. On Optimal, Minimal BRDF Sampling for Reflectance Acquisition. ACM Trans. Graph. 34, 6, Article 186 (Oct. 2015), 11 pages. https://doi.org/10.1145/2816795.2818085

Matt Pharr and Greg Humphreys. 2010. Physically Based Rendering, Second Edition: From Theory To Implementation (2nd ed.). Morgan Kaufmann Publishers Inc., San Francisco, CA, USA.

Romain Vergne, Romain Pacanowski, Pascal Barla, Xavier Granier, and Christophe Schlick. 2009. Light Warping for Enhanced Surface Depiction. ACM Trans. Graph. 28, 3, Article 25 (July 2009), 8 pages. https://doi.org/10.1145/1531326.1531331 